\title{
Optimal Scheduling of Logistical Support for Medical Resource with Demand Information Updating
}

\author{
Ming Liu and Yihong Xiao \\ Department of Management Science and Engineering, Nanjing University of Science and Technology, Nanjing 210094, China \\ Correspondence should be addressed to Ming Liu; liumingseu@126.com
}

Received 1 April 2014; Revised 5 August 2014; Accepted 5 August 2014

Academic Editor: Michael Lütjen

Copyright ( 2015 M. Liu and Y. Xiao. This is an open access article distributed under the Creative Commons Attribution License, which permits unrestricted use, distribution, and reproduction in any medium, provided the original work is properly cited.

\begin{abstract}
This paper presents a discrete time-space network model for a dynamic resource allocation problem following an epidemic outbreak in a region. It couples a forecasting mechanism for dynamic demand of medical resource based on an epidemic diffusion model and a multistage programming model for optimal allocation and transport of such resource. At each stage, the linear programming solves for a cost minimizing resource allocation solution subject to a time-varying demand that is forecasted by a recursion model. The rationale that the medical resource allocated in early periods will take effect in subduing the spread of epidemic and thus impact the demand in later periods has been incorporated in such recursion model. A custom genetic algorithm is adopted to solve the proposed model, and a numerical example is presented for sensitivity analysis of the parameters. We compare the proposed medical resource allocation mode with two traditional operation modes in practice and find that our model is superior to any of them in less waste of resource and less logistic cost. The results may provide some practical guidelines for a decision-maker who is in charge of medical resource allocation in an epidemics control effort.
\end{abstract}

\section{Introduction}

Over the past few years, the world has grown increasingly concerned about the threat of different epidemics. Disastrous epidemic events such as SARS and H1N1 significantly impacted people's life. The outbreak of infections in Europe is another recent example. The infection, from a strain of Escherichia coli, can lead to kidney failure and death and is difficult to treat with antibiotics. It is now widely recognized that a large-scale epidemic diffusion can conceivably cause many deaths and more people of permanent sequela, which presents a severe challenge to local or regional healthcare systems.

After an epidemic outbreak, public officials are faced with many critical issues, the most important of which being how to ensure the availability and supply of medical resource so that the loss of life may be minimized and the rescue operation efficiency maximized. The medicine logistics in an epidemics controlling system is often complex and difficult. $\mathrm{Hu}$ et al. [1] compared public-health management mechanisms in both USA and China from the following three aspects, organizational structure, management system, and logistics network, and pointed out some deficient areas in the Chinese public-health management mechanism. To date, medicine logistics operation in epidemic control activities in China has traditionally been done unsystematically and separately, based on the decision-makers' experience and disregarding the interrelationship between the time-varying demand and the logistics operation planning from a systematic perspective. Thus, this paramount life-saving and costly logistics problem opens up a wide range of applications of Operations Research/Management Science techniques and has motivated many recent research works.

In this paper, a time-space network model for the medical resource allocation problem in controlling epidemic diffusion is proposed. It couples a forecasting mechanism for the dynamic demand of the medical resource based on the epidemic diffusion pattern of susceptible-exposed-infectedrecovered (SEIR) model [2] and a multistage programming model for optimal allocation and transport of such resource. The two dynamic processes are woven together and interactively proceed to model the epidemic diffusion and the medical resource allocation. Particularly, given the dynamic demand for the medical resource at each stage predicted by the forecasting mechanism, the linear programming problem 
solves for the cost minimizing resource allocation pattern subject to related operating constraints. The optimal solution of the resource allocation will then determine their availability at each emergent district hospital, upon which the efficiency of rescuing effort is conditioned (assuming the other needed healthcare technologies and human resource are guaranteed). The efficiency of the rescuing effort will determine the recovering rate of the infected population, which, in turn, will generate the new forecast of the demand for medical resource by updating the SEIR diffusion model. The above described model is expected to be an effective decision-making tool that can help improve the efficiency of medicine logistics when an epidemic outbreaks. To the best of our knowledge, the dynamic and interactive optimization process has never been reported in the existing works.

The remainder of the paper is organized as follows: Section 2 is the literature review. Section 3 introduces the time-space network model, which combines a time-varying demand forecast model based on the epidemic diffusion rule, and a multistage programming model for cost minimizing allocation of the medical resource. The solution procedure for the optimization model is proposed in Section 4. A numerical example and a short sensitivity analysis are presented in Section 5. Finally, Section 6 discusses the limitations of the proposed approach and suggests future research directions.

\section{Literature Review}

Considering the relationship between the epidemic diffusion and the associated medical resource allocation, we review two streams of recent research efforts here: one is focused on the epidemic diffusion modeling and the other is related to the medical resource allocation modeling.

2.1. Epidemic Diffusion Modeling. Most analytical works on epidemic diffusion are concentrated on the compartmental epidemic models described by ordinary differential equations [3-5]. In these models, the total population is divided into several classes and each class of people is closed into a compartment. The sizes of the compartments are assumed to be large enough and the mixing of members to be homogeneous.

The second stream of research is on the development of epidemic diffusion models by applying complex network theory to the traditional compartment models [6-8]. Recently, Jung et al. [9] extended the previous studies on the prevention of the pandemic influenza to evaluate time-dependent optimal prevention policies, and they found that the quarantine policy was very important and more effective than the elimination policy, during the disease spread. Wang et al. [10] presented some suggestions for the epidemic prevention and infection control in the Wenchuan earthquake areas, Sichuan Province, China.

The third stream of research is on the development of epidemic diffusion models by applying simulation methods, including computer simulation and numerical computation [11-13]. For example, Samsuzzoha et al. [14] used a diffusive epidemic model to describe the transmission of influenza. The equations were solved numerically by using the splitting method under different initial distribution of population density. Further, Samsuzzoha et al. [15] presented a vaccinated diffusive compartmental epidemic model to explore the impact of vaccination as well as diffusion on the transmission dynamics of influenza.

Recently and importantly, a robust data-driven fault detection approach is proposed with application to a wind turbine benchmark [16]. The main challenges of the wind turbine fault detection lie in its nonlinearity, unknown disturbances, and significant measurement noise. Sometimes the relative data may be missed $[17,18]$. These works are constructive and helpful to understand and model the epidemic diffusion process in a very different way.

The above mentioned works represent some of the research on various differential equation models for epidemic diffusion and control. Although the emphasis of this paper is on the efficient allocation of medical resource, a basic component of our model, the forecasting mechanism for their dynamic demand, utilizes one of such epidemic diffusion models.

2.2. Medical Resource Allocation Modeling. To the best of our knowledge, a great deal of researches has been published with the topic on optimal allocation of medical resource [1923]. To optimize the process of materials distribution in an epidemic diffusion system and to improve the distribution timeliness, Liu and Zhao [24] modeled the emergency materials distribution problem as a multiple traveling salesman problem with time window. Wang et al. [25] constructed a multiobjective stochastic programming model with timevarying demand for the emergency logistics network based on the epidemic diffusion rule. A genetic algorithm coupled with Monte Carlo simulation was adopted to solve the optimization model. Qiang and Nagurney [26] proposed a humanitarian logistic model for supply/distribution of critical needs in a disruption caused by a natural disaster. They considered a general network structure and disruptions that may have an impact on both network link capacities and product demand. The problem was studied in a bicriteria system optimization framework for network performance. Recently, Rachaniotis et al. [27] presented a deterministic resource scheduling model in epidemic control. In their work, a deterministic model, appropriate for large populations, where random interactions could be averaged out, was used for the epidemic's rate of spread. Besides, a case of the mass vaccination against $\mathrm{H} 1 \mathrm{~N} 1$ influenza in the Attica region, Greece, and a comparative study of the model's performance versus the applied random practice were presented.

To deal with the complexity and difficulty in solving the medical resource allocation problem, we observe a trend in solution methodologies, that is, decomposing the original problem, which can be a multicommodity, multimodal, or multiperiod model, into several mutually correlated subproblems, and then solve them systematically in same decision scheme. For instance, Barbarosolu et al. [28] proposed a bilevel hierarchical decomposition approach for helicopter mission planning during a disaster relief operation. The top-level model was formulated to deal with the tactical decisions, covering the issues of helicopter fleet management, crew assignment, and the number of tours undertaken by 


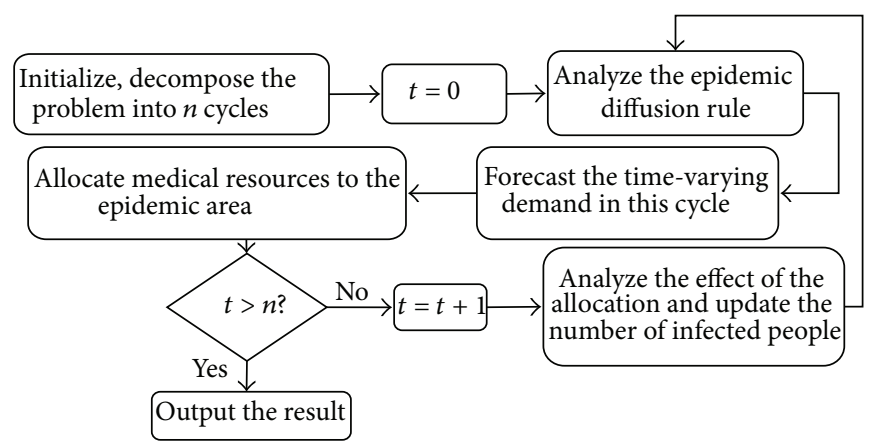

FIGURE 1: Operational procedure of the dynamic medicine logistics network.

each helicopter. The base-level model aimed to address the corresponding operational decisions, including routing, loading/unloading, and refueling scheduling. References [25, 29] were more recent works following this line.

Furthermore, we note that most of the previous works were carried out under the assumption that the relief demand is not time sensitive. While in reality, the demand for medical resource is dynamic, and the medical resource allocated in early cycles will affect the demand in later periods. In this paper, we will use a discrete time-space network to model the medical resource allocation problem when an epidemic outbreaks. In each decision cycle, the problem is constructed as a linear programming model to solve for the cost minimizing allocation solution subject to the time-varying demand that is predicted by the epidemic diffusion rule. As such, this paper attempts to bridge the two streams of literature, the epidemic diffusion and the medicine logistics, which were studied separately in existing literature.

\section{The Mathematical Model}

Epidemic diffusion process can be divided according to its development into three stages [30]. The first stage is the inception of the epidemic in very limited population, which if noticed in time and treated properly can be controlled effectively without causing a wide spread. In the second stage, the epidemic has broken out into a widespread diffusion. An important part of epidemic control and rescue campaign is to ensure the timely delivery of the needed medical resource according to the dynamic demand as determined by the progress of the epidemic spread. In the third stage, the epidemic diffusion has been controlled and the demand for medical resource has significantly declined. Liu et al. [31] proposed a model for studying medical resource distribution in the first stage. In this paper, we will concentrate on the logistics problem of medical resource allocation in the second stage. Particularly, we will study how the area distribution centers (ADC) should supply the district distribution centers (DDC) and how the DDCs should deliver the needed medical resource to the emergency designated hospitals $(\mathrm{EDH})$ in the most efficient and cost-effective way. Here we assume there are several ADCs in the epidemic spread area, which can be divided into several municipal districts or towns. Each district will have one or more DDCs which supply the needed medical resource to the EDHs in that district.

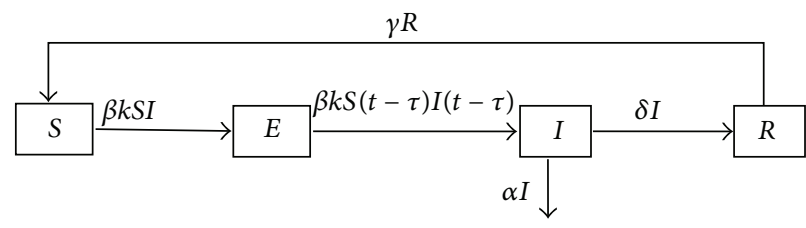

FIGURE 2: SEIRS model based on a small-world network.

Since demand from each EDH is determined by the number of patients hospitalized there and varies according to the progress of epidemic diffusion, the allocation of medical resource need to chase the demand over time. Figure 1 below gives a diagram of operations outlining the execution of the proposed model. The sequential operational routine continues until the epidemic diffusion gets under control. As Figure 1 shows, medical resource allocation process is decomposed into $n$ decision-making cycles. Each decision-making cycle includes three phases: epidemic diffusion analysis, demand forecasting, and medical resource allocation. These three phases are executed iteratively. The effect of the medical resource allocation is analyzed, and the number of infected people is updated at each cycle during the entire distribution process.

In the sequel, we will introduce SIERS model, a wellrecognized epidemic diffusion model, in Section 3.1, propose a forecasting model for the dynamic demand for the medical resource during the epidemic diffusion in Section 3.2, and a linear programming model for distribution of medical resource according to the forecasted dynamic demand.

3.1. SEIRS Epidemic Diffusion Model. The SEIR model has been widely adopted by researchers to study epidemic diffusion. It is based on small-world network theory and provides a good match to the actual social network [2]. Generally, the total population is divided into four classes, susceptible people $(S)$, exposed people $(E)$, infected people $(I)$, and recovered people $(R)$, and each class of people is closed into a compartment. Tham [32] showed that some of the recovered people who were discharged from hospitals might be reinfected. Figure 2 shows, without consideration of migration, the natural birth rate and death rate of the population, the epidemic process can be described by a SEIRS model based on a small-world network [25]. 
The dynamic system for the SEIRS diffusion model can be rewritten by the following ordinary differential equations:

$$
\begin{aligned}
& \frac{d S}{d t}=-\beta k S(t) I(t)+\gamma R(t), \\
& \frac{d E}{d t}=\beta k S(t) I(t)-\beta k S(t-\tau) I(t-\tau), \\
& \frac{d I}{d t}=\beta k S(t-\tau) I(t-\tau)-(\alpha+\delta) I(t), \\
& \frac{d R}{d t}=\delta I(t)-\gamma R(t) .
\end{aligned}
$$

In the above system of equations, $S(t), E(t), I(t)$, and $R(t)$ represent, respectively, the number of susceptible people, the number of exposed people, the number of infected people, and the number of recovered people. $k$ is the average degree of distribution for this small-world network, which can be interpreted as the average contact number of susceptible people of each infected person; $\beta$ is the propagation coefficient of the epidemic; $\gamma$ is the rate of the recovered people who are not immune and thus may be reinfected; $\delta$ is the recovery rate; $\alpha$ is the death rate; $\tau$ represents the incubation period of the disease. Consider $k, \beta, \gamma, \delta, \alpha, \tau>0$.

ODE (1) states the following: (i) the growth rate of the susceptible population is determined by the returning population who are recovered but not immune and the losing population who actually get exposed to the disease and thus are counted towards the class of $E(t)$. The latter is in proportion to the propagation coefficient $\beta$, the average contact number of susceptible people of each infected person, $k$, and both of the current mass of the susceptible population and the current mass of the infected population. (ii) The growth rate of the exposed population is determined by the difference between the entering population, those of susceptible people who actually get exposed to the disease, and the exiting population, those of exposed population who get sick after the incubation period of the disease; (iii) the growth rate of the infected population is determined by the difference between the entering population, those of exposed population who get sick, and the exiting population who are either recovered or dead; and, finally (iv) the growth rate of the recovered population is determined by the difference between the joining population of the newly recovered and the losing population of the reinfected people.

Particularly, as we noted in (iii), the number of infected people, $I(t)$, is determined by the population of the recovered people and the onset exposed people at the end of the incubation period. Hence, improving the recovery rate, $\delta$, and reducing the propagation coefficient, $\beta$, are the two effective measures to take in suppressing the growth of $I(t)$. In the context of epidemic controlling operation, that means sufficient medical resource should be allocated to the emergent designated hospitals (EDH).

\subsection{The Forecasting Model for the Time-Varying Demand.} Demand for medical resource has been studied in a variety of forms in the literature, such as a time-varying value [33] or obeying some stochastic distribution [29]. However,

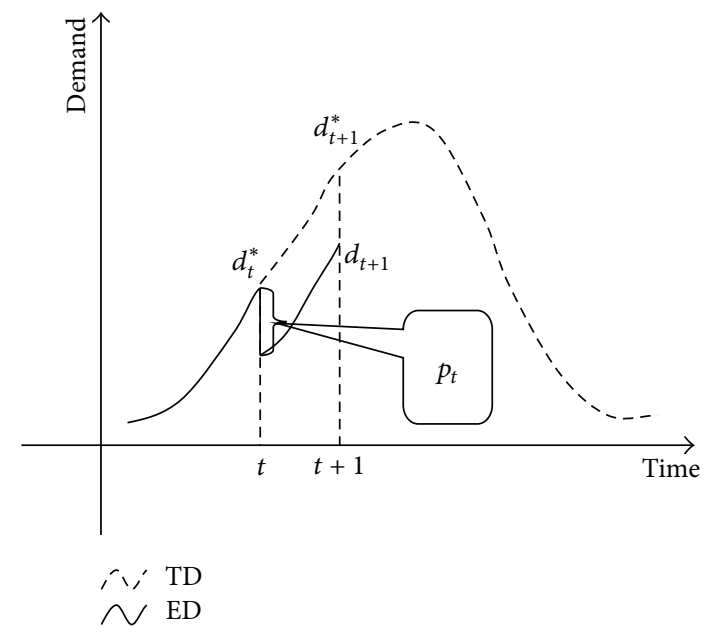

FIGURE 3: Diagrammatic sketch of the time-varying demand.

the impact of earlier resource allocation to the demand in later periods has basically been ignored in these approaches.

To address this deficiency, we propose the following linear relationship between the demand for medical resource and the number of infected people at time $t$ based on the SEIRS epidemic diffusion model:

$$
d_{t}=a I(t)
$$

where $d_{t}$ refers to the demand for medical resource at time $t$ and $a$ is the proportionality coefficient. In our interviews with the public-healthcare administrative personnel about controlling the epidemic spread, we found this linear forecasting function is the one they commonly adopted. Here we define it as the traditional demand (TD). However, a lag effect of earlier medicine allocation should be taken into account in the current demand forecast. As shown in Figure 3, the horizontal axis represents the decision-making cycle, and the vertical axis stands for demand in an epidemic area. The dotted line is a trajectory of (2), and the solid curve is the expected demand (ED). For instance, if the demand for medical resource at cycle $t$ is $d_{t}^{*}$, and according to (2), the demand at cycle $t+1$ would have been $d_{t+1}^{*}$. However, a certain amount of medical resource, $p_{t}$, had been allocated to the disaster area during cycle $t$, and it would be taking effect in cycle $t+1$ in curing the infected patients in hospitals and thus subduing the diffusion. Hence, the expected demand for medical resource at cycle $t+1$ should be $d_{t+1}$, instead of $d_{t+1}^{*}$.

The following growth factor is introduced by the above observation to account for the lag effect:

$$
\eta_{t}=\frac{d_{t+1}^{*}-d_{t}^{*}}{d_{t}^{*}}
$$

Herein, the growth factor $\eta_{t}$ can be either positive (increasing demand) or negative (decreasing demand) and may vary in different cycles for the different demand $d_{t}^{*}$. As mentioned before, part of the recovered people who are discharged from the healthcare department may be reinfected. Thus, we define the effective cure rate as $\theta$ as the percent 


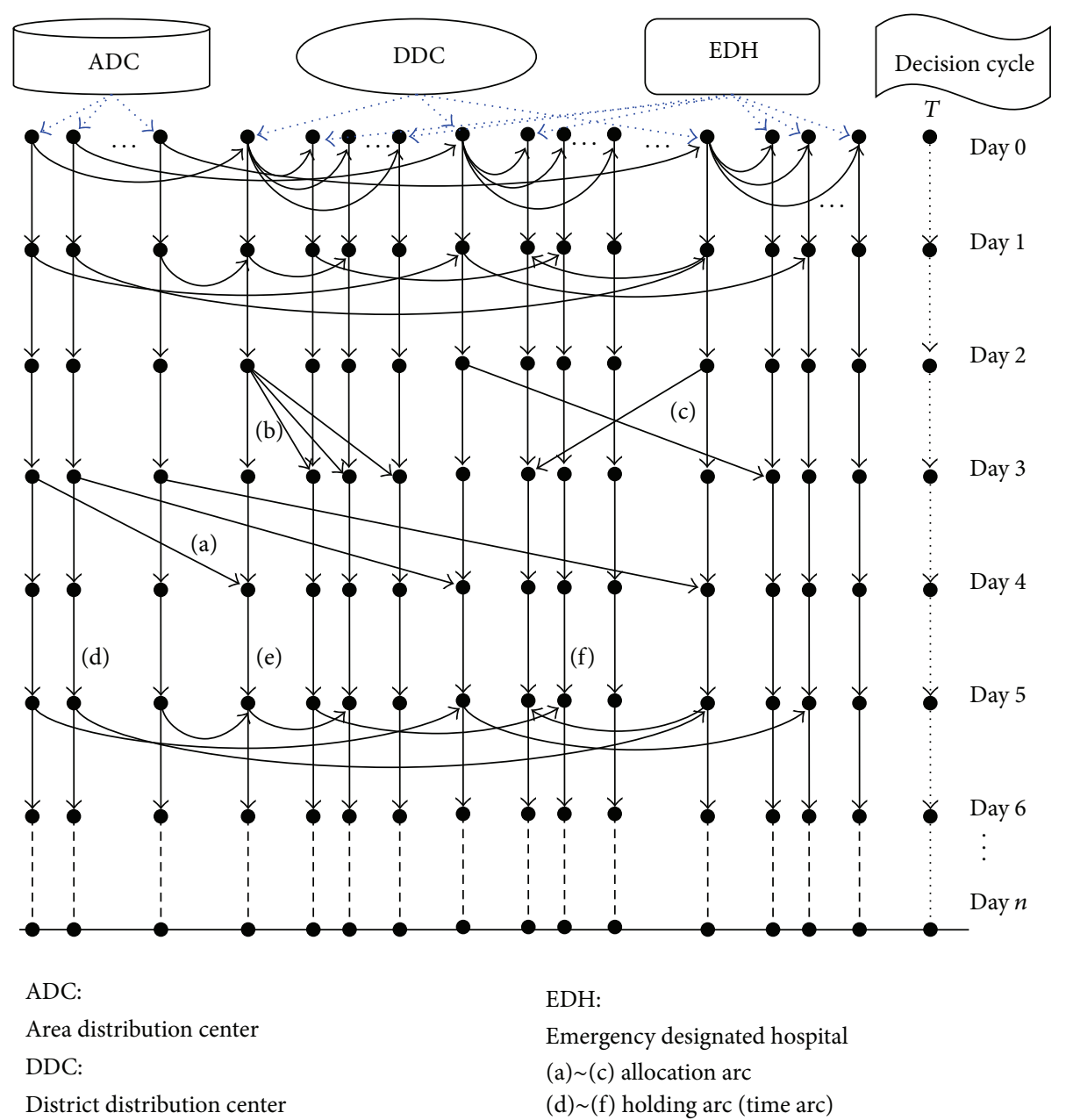

FIgURE 4: Time-space network of medical resource allocation.

of recovered people who are not reinfected. Considering that each infected person needs a period of time to receive treatment and get cured, herein we denote the treatment cycle as $\Gamma$ and we assume it to be an integral multiple of the decision cycle. Then, the commuted effective cure rate in each decision cycle can be obtained as $\theta / \Gamma$. Such an assumption would be feasible if the decision cycle is small enough, for example, one day. Hence, it helps us get the following recursion formulas:

$$
\text { when } t=1, \quad d_{1}=\left(1+\eta_{0}\right)\left(1-\frac{\theta}{\Gamma}\right) d_{0} \text {; }
$$

when $t=2, \quad d_{2}=\left(1+\eta_{1}\right)\left(1-\frac{\theta}{\Gamma}\right) d_{1}$

$$
=\left(1+\eta_{0}\right)\left(1+\eta_{1}\right)\left(1-\frac{\theta}{\Gamma}\right)^{2} d_{0}
$$

when $t=n, \quad d_{n}=\prod_{i=0}^{n-1}\left(1+\eta_{i}\right)\left(1-\frac{\theta}{\Gamma}\right)^{n} d_{0}$.
Herein, $\prod_{i=0}^{n-1}\left(1+\eta_{i}\right)=\left(1+\eta_{0}\right)\left(1+\eta_{1}\right) \cdots\left(1+\eta_{n-1}\right)$. $d_{0}=a I(0)$ is the initial demand for medical resource in the epidemic area, and $I(0)$ is the initial number of infected people in the epidemic area. Recursion formulas (4) are our prescribed forecast model for the demand of medical resource. In what follows, we will propose a medicine logistics operation model to minimize the total allocation cost based on the forecasting model.

3.3. Time-Space Network of the Medicine Logistics. In this subsection, a multistage programming model for cost minimizing allocation of the medical resource is built upon a time-space network. Figure 4 is the schematic diagram of the network. The vertical axis represents the time duration. The horizontal axis represents the area distribution center (ADC), the district distribution center (DDC), and the emergency designated hospital (EDH), respectively. The allocation arcs are defined as follows: (a) represents that medical resource is transported from ADC to DDC; (b) stands for that medical resource is allocated from $\mathrm{DDC}$ to $\mathrm{EDH}$ in the same district; (c) refers to that medical resource is allocated from DDC to $\mathrm{EDH}$ in the other district; (d) (f) are time duration arcs for different departments. 
3.3.1. Assumptions. The following assumptions are needed to facilitate the model formulation in the following sections.

(1) In the event of an epidemic outbreak, it is paramount for the government and the entire society to control the spread and rescue the infected. Thus it is reasonable to assume that the government can ensure the adequate supply of the needed medical resource either from domestic pharmaceutical companies or imported. Hence, there is enough medical resource in ADCs during the entire operation process.

(2) Once an epidemic outbreak, the government will take strict control measures so that each epidemic area can be isolated from other areas to avoid the cross spread of the disease. In each epidemic area, the government will appoint a hospital to be the $\mathrm{EDH}$, to be responsible for the rescue work in such an isolated area.

(3) Medical resource in this paper is an assembled product, which may include water, vaccine, antibiotic, and so forth.

3.3.2. Notations. Notations used in the following programming model are specified as follows:

$c c_{i j}$ : unit transportation cost of medical resource from $\mathrm{ADC} i$ to $\mathrm{DDC} j$,

$c r_{i j}$ : unit transportation cost of medical resource from $\mathrm{DDC} i$ to $\mathrm{EDH} j$,

$e s_{i t}$ : the available quantity of medical resource in ADC $i$ in decision cycle $t$,

$z r_{i t}$ : quantity of medical resource allocated to DDC $i$ in decision cycle $t$,

$x_{i j t}$ : medical resource transported from $\operatorname{ADC} i$ to DDC $j$ in decision cycle $t$,

$y_{i j t}$ : medical resource transported from DDC $i$ to $\mathrm{EDH} j$ in decision cycle $t$,

$d_{i t}$ : demand for medical resource in $\mathrm{EDH} i$ in decision cycle $t$,

$T$ : set of decision cycles,

$C$ : set of $\mathrm{ADCs}$,

$R:$ set of DDCs,

$H$ : set of EDHs.

3.3.3. Model Formulation. Let $F(x, y)$ be the objective function of the total cost of medical resource allocation. Based on the above assumptions and descriptions, the proposed problem can be formulated as follows:

$$
\begin{array}{ll}
\text { Min } & F(x, y)=\sum_{t \in T} \sum_{i \in C} \sum_{j \in R} x_{i j t} c c_{i j}+\sum_{t \in T} \sum_{i \in R} \sum_{j \in H} y_{i j t} c r_{i j} \\
\text { s.t. } \quad & \sum_{i \in C} x_{i j t}=z r_{j t}, \quad \forall j \in R, t \in T \\
& \sum_{j \in R} x_{i j t} \leq e s_{i t}, \quad \forall i \in C, t \in T \\
& \sum_{i \in R} y_{i j t}=d_{j t}, \quad \forall j \in H, t \in T \\
& \sum_{j \in H} y_{i j t} \leq z r_{i t}, \quad \forall i \in R, t \in T \\
& d_{i 0}=a I_{i}(0), \quad \forall i \in H \\
& d_{i t}=\prod_{t=0}^{t-1}\left(1+\eta_{i t}\right)\left(1-\frac{\theta}{\Gamma}\right)^{t} d_{i 0}, \\
& \forall i \in H, t \in\{T, t \neq 0\} \\
x_{i j t} \geq 0, \quad \forall i \in C, \quad j \in R, t \in T \\
y_{i j t} \geq 0, \quad \forall i \in R, \quad j \in H, t \in T .
\end{array}
$$

In this optimization model, $x_{i j t}$ and $y_{i j t}$ are the decision variables. The objective function (5) is to minimize the total cost of medical resource allocation, which is the transportation cost for delivering the medical resource from ADCs to DDCs and from DDCs to EDHs. Constraints (6) (9) are the flow conservation equations. Particularly, constraint (6) suggests that each DDC can obtain medical resource from all ADCs. Constraint (7) ensures that the total shipments from any ADC cannot exceed the available amount of the resource in this ADC. Constraint (8) states that the period demand generated by the forecasting model in Section 3.2 at each EDH must be satisfied. That is, the shipments from all DDCs to each EDH must be equal to the demand at this EDH. Constraint (9) implies that the total shipments from any DDC cannot exceed the available stock in this DDC. Constraints (10)-(11) are forecasting model for the time-varying demand (Section 3.2). Herein, $\eta_{i t}$ is the growth factor (can be either positive or negative) of the demand for medical resource in EDH $i$ in decision cycle $t$. Finally, (12) and (13) are the nonnegativity of the flows. Such model is a dynamic and multistage programming model.

\section{Solution Methodology}

To solve the above optimization model, (10)-(12) are adopted to calculate the time-varying demand firstly. After that, to $\forall t \in T$, the research model can be converted as a twostage linear programming model. The feature of such a twostage programming problem is that both the input quantity and the output quantity of the medical resource in the 
TABLE 1: Values of parameters in the SEIRS model.

\begin{tabular}{|c|c|c|c|c|c|c|c|c|}
\hline & \multicolumn{4}{|c|}{$\mathrm{ADC} 1$} & \multicolumn{4}{|c|}{$\mathrm{ADC} 2$} \\
\hline & \multicolumn{2}{|c|}{ DDC 1} & \multicolumn{2}{|c|}{ DDC 2} & \multicolumn{2}{|c|}{ DDC 3} & \multicolumn{2}{|c|}{ DDC 4} \\
\hline & EDH 1 & EDH 2 & EDH 3 & EDH 4 & EDH 5 & EDH 6 & EDH 7 & EDH 8 \\
\hline$S(0)$ & $5 \times 10^{3}$ & $4.5 \times 10^{3}$ & $5.5 \times 10^{3}$ & $5 \times 10^{3}$ & $6 \times 10^{3}$ & $4.8 \times 10^{3}$ & $5.2 \times 10^{3}$ & $4 \times 10^{3}$ \\
\hline$E(0)$ & 30 & 35 & 30 & 40 & 25 & 40 & 50 & 45 \\
\hline$I(0)$ & 5 & 6 & 7 & 8 & 4 & 7 & 9 & 10 \\
\hline$R(0)$ & 0 & & & & & & & \\
\hline$\beta$ & $5 \times 10^{-5}$ & & & & & & & \\
\hline$\langle k\rangle$ & 6 & & & & & & & \\
\hline$\delta$ & 0.3 & & & & & & & \\
\hline$d$ & $1 \times 10^{-3}$ & & & & & & & \\
\hline$\gamma$ & $1 \times 10^{-3}$ & & & & & & & \\
\hline$\tau$ & 5 & & & & & & & \\
\hline
\end{tabular}

DDCs are unknown. There are many available techniques for solving such a problem, and a genetic algorithm is commonly used. Hence, a genetic algorithm coupled with MATLAB 7.0 mathematical programming solver is adopted to solve the model.

4.1. Chromosome Coding and Population Initializing. The first step of a genetic algorithm is to define the coding method of the chromosome. As is well known, the real number coding is superior to the binary coding in both aspects of quality and efficiency of the solution. Besides, the real number coding is closer to the actual problem findings and easier to interpret in the real world problem. Herein, the real number coding is adopted. For $\forall t \in T$, each chromosome contains $R$ bit gene, where $R$ is the number of DDC. The value of each bit refers to the available amount of medical resource in each DDC, which is also the quantity of medical resource replenished from all ADCs. Each individual in the initial population is generated by a random method, subject to the related resource constraints in the programming model.

4.2. Fitness Definition. The fitness of each individual is obtained by computing the objective function

$$
F(x, y)=\sum_{t \in T} \sum_{i \in C} \sum_{j \in R} x_{i j t} c c_{i j}+\sum_{t \in T} \sum_{i \in R} \sum_{j \in H} y_{i j t} c r_{i j} .
$$

Herein, the fitness function contains two parts. The first part is the total transportation cost between ADCs and DDCs. The second part is the total transportation cost between DDCs and EDHs. Obviously, the lower the total cost is, the better the fitness of the individual is.

4.3. Selection Operator. The best individual copy strategy is adopted in selection section. That means, each time when selection operator is iterated, the worst chromosome in the population will be replaced by the best one.

4.4. Crossover Operator. A crossover operator is one of the most important operators in a genetic algorithm. Different crossover operators are suitable for different kinds of chromosomes. According to the real number coding in this paper, an arithmetic crossover is adopted. Let $P_{1}$ and $P_{2}$ represent the two parent chromosomes, and $P_{c 1}$ and $P_{c 2}$ stand for the two children chromosomes, respectively. The linear relationship between the parent and the children chromosomes can be formulated as follows:

$$
\begin{aligned}
& P_{c 1}=\mu P_{1}+(1-\mu) P_{2}, \\
& P_{c 2}=(1-\mu) P_{1}+\mu P_{2} .
\end{aligned}
$$

Herein, $\mu=U(0,1)$ is a uniform random number between 0 and 1 . Note that both of these two children chromosomes automatically satisfy the resource constraints in the multistage programming model. The range of the crossover probability $p_{c}$ is $0.2 \sim 0.8$.

4.5. Mutation Operator. A mutation operator is intended to simulate genetic mutation during biological evolution. Mutation is operated on some bits of individuals at a probability of $p_{m}$. This probability is generally very small and is set in the range $0.001 \leq p_{m} \leq 0.1$. When mutating, we exchange a pair of genes in the individual.

4.6. Termination Condition. As the optimal result is unpredictable, a max iteration is given for the termination.

\section{Numerical Tests}

5.1. A Numerical Example. We present a numerical example to illustrate the efficiency of the proposed model. Assume there is a smallpox outbreak in a city, which has two ADCs and four DDCs. Two hospitals are designated in each district, and each EDH can service a certain amount of patients. The values of the parameters in the epidemic diffusion model are given in Table 1.

Figure 5 depicts a numerical simulation of the epidemic model at EDH1 in this effected region. The four curves, respectively, represent the number of four groups of people $(S, E, I, R)$ over time. As mentioned in Section 3, the process of epidemic diffusion is divided into three stages and our work in this paper is focused on the second stage. According to Figure 5, such a stage can be ranged from the 10th day 


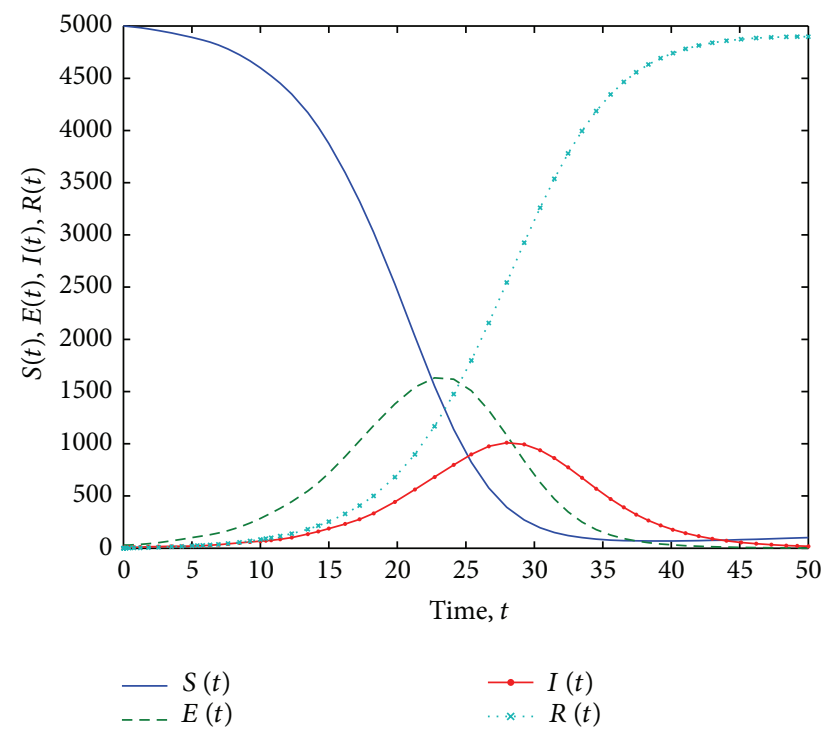

FIGURE 5: Solution of the SEIRS epidemic diffusion model (EDH1).

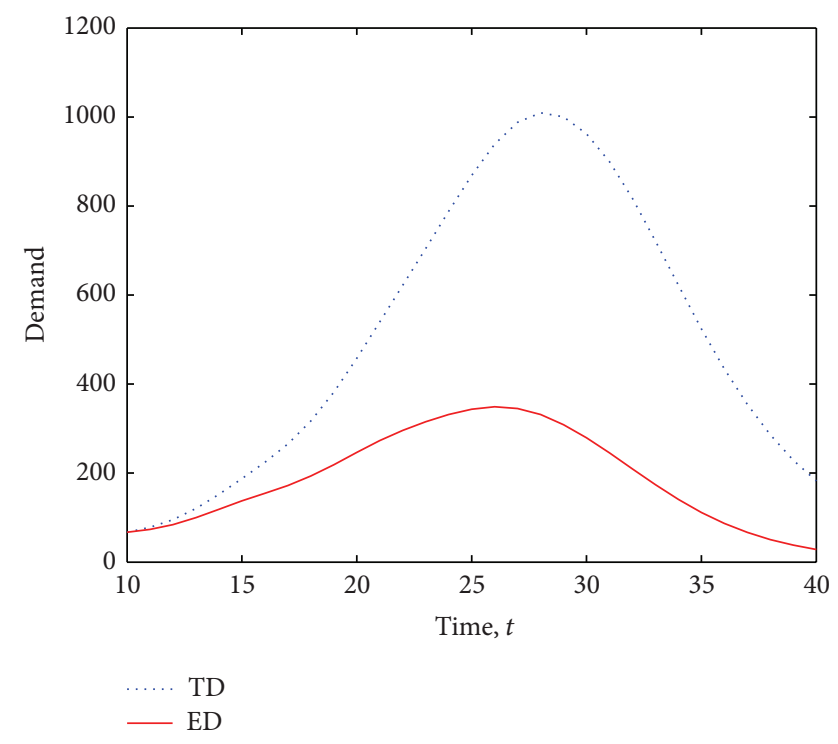

Figure 6: Demand in EDH1 by the two different methods.

(decision-making cycle $t=0$ ) to the 40th day (decisionmaking cycle $t=30$ ). Of course, when different emergency outbreak happens, the result can be adjusted correspondingly.

To facilitate the calculate process in the following sections, the decision-making cycle is assumed to be one day. Let $a=1$; MATLAB 7.0 mathematical programming solver coupled with (1) and (2) is adopted to calculate the TD for medical resource in each EDH. Furthermore, given that $\theta=90 \%$ and $\Gamma=15$ (days), the growth factor $\eta_{i t}$ in each decision-making cycle can be obtained. Then, the ED for medical resource in each $\mathrm{EDH}$ in each decision cycle can be forecasted according to (12)-(13). Taking EDH1 as an example, the demand for medical resource in each decision-making cycle by these two different methods is compared as shown in Figure 6.

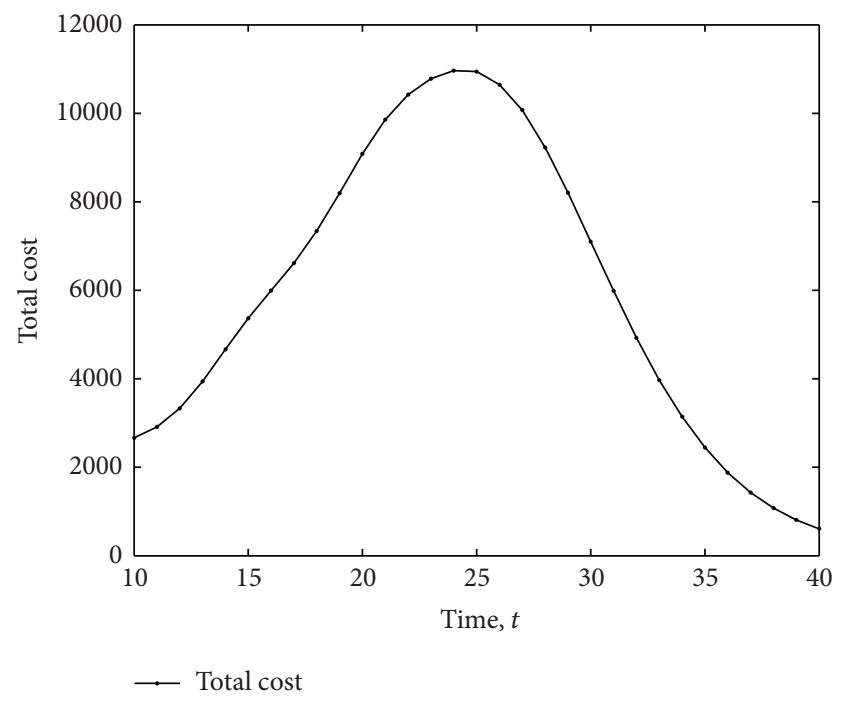

FIGURE 7: Total cost in each decision-making cycle (Unit: \$).

One can observe in Figure 6 that ED is way below TD, suggesting that the allocation of medical resource in the early periods will significantly reduce the demand in the following periods. The second observation is that both curves exhibit similar trends; namely, the demand will first increase along with the spreading the epidemic and then will decrease after the epidemic is brought under control.

We now proceed to illustrate the optimal allocation of the resource at each $\mathrm{EDH}$. Table 2 shows the unit operation cost of medicine between two different departments.

Let $n=200, p_{c}=0.75$, and $p_{m}=0.01$. The algorithm is set to terminate in 200 generations. Taking the allocation at cycle $t=0$ as the initial example, we solve the above programming model (7)-(15) according to the solution procedure. The convergent allocation scheme is reported in Table 3 and the total operation cost is 2663.22 .

To test the accuracy and stability of the algorithm, the computation process has been repeated for six times independently. As shown in Table 4, the convergent results in these six times are very close and the deviation is less than $0.065 \%$. This proves the proposed algorithm is stable and accurate. We execute the solution procedure (Table 1) to find the dynamic allocation result of medical resource and show in Figure 7 the total cost at each decision-making cycle.

Comparing Figure 7 with Figure 6, one can find that the curve of the total operation cost matches well the demand curves in their variation pattern, suggesting that the cost of medical resource allocation mainly depends on the demand. The characteristics also reflect the hysteresis effect in an epidemic controlling system that medicine logistics lags behind the epidemic diffusion.

In next subsection, we will compare the proposed model with the two traditional allocation measurements that have been used in practice.

5.2. Model Comparison. Based on our interviews with the public healthcare administrative personnel in China, there are two traditional measurements in practice to predict 
TABLE 2: Unit operation cost between two different departments (Unit: \$).

\begin{tabular}{lcccccccccc}
\hline Cost & ADC1 & ADC2 & EDH1 & EDH2 & EDH3 & EDH4 & EDH5 & EDH6 & EDH7 & EDH8 \\
\hline DDC1 & 3.5 & 2 & 1 & 2 & 4 & 2.5 & 5 & 5 & 2.5 & 1.5 \\
DDC2 & 1.5 & 2 & 2 & 2.5 & 2 & 3 & 5 & 4 & 2.5 & 2 \\
DDC3 & 3 & 1.5 & 2.5 & 3 & 5 & 2 & 1 & 3 & 1.5 \\
DDC4 & 2.5 & 3 & 4 & 4 & 1.5 & 2.5 & 3 & 2 & 2 & 1.5 \\
\hline
\end{tabular}

TABLE 3: Medical resource allocation result at decision-making cycle $t=0$ (Unit: \$).

\begin{tabular}{|c|c|c|c|c|c|c|c|c|}
\hline & \multicolumn{2}{|c|}{$\mathrm{DDC1}$} & \multicolumn{2}{|c|}{ DDC2 } & \multicolumn{2}{|c|}{ DDC3 } & \multicolumn{2}{|c|}{ DDC4 } \\
\hline $\mathrm{ADC1}$ & \multicolumn{2}{|c|}{0} & \multicolumn{2}{|c|}{186.6901} & \multicolumn{2}{|c|}{0} & \multicolumn{2}{|c|}{163.759} \\
\hline \multirow[t]{2}{*}{$\mathrm{ADC} 2$} & \multicolumn{2}{|c|}{188.8323} & \multicolumn{2}{|c|}{0} & \multicolumn{2}{|c|}{191.9134} & \multicolumn{2}{|c|}{0} \\
\hline & EDH1 & EDH2 & EDH3 & EDH4 & EDH5 & EDH6 & EDH7 & EDH8 \\
\hline DDC1 & 67.1588 & 43.0695 & 0 & 31.1464 & 0 & 0 & 0 & 47.4576 \\
\hline DDC2 & 0 & 23.5330 & 82.9355 & 22.1931 & 0 & 0 & 31.4635 & 26.5650 \\
\hline DDC3 & 0 & 0 & 0 & 38.6671 & 74.9403 & 0 & 78.3061 & 0 \\
\hline DDC4 & 0 & 0 & 28.1169 & 14.6546 & 0 & 86.9697 & 18.6328 & 15.3849 \\
\hline
\end{tabular}

TABLE 4: Total cost in cycle $t=0$ (Unit: \$).

\begin{tabular}{lcccccc}
\hline Run & 1 & 2 & 3 & 4 & 5 & 6 \\
\hline $\begin{array}{l}\text { Total } \\
\text { cost }\end{array}$ & 2664.97 & 2663.22 & 2663.22 & 2663.22 & 2664.97 & 2663.22 \\
\hline
\end{tabular}

the demand for medical resource in case of an epidemic outbreak. Both of them utilize (2) as the basic forecasting method. In the first traditional measurement, referred as Traditional 1, the medical resource will only be allocated through administrative distribution. That is, an ADC will only service the DDCs in its own area, and a DDC will only service the EDHs in its own district. For instance, as Table 1 shows, ADC 1 will only service DDC1 and DDC2, and DDC1 will only replenish medical resource to EDH1 and EDH2. The second traditional measurement, referred here as Traditional 2 , is based on the same forecasting method of (2) but allows cross area distribution. The total costs of these three different models are compared and illustrated in Figure 8.

Several interesting observations can be drawn from Figure 8 . First, the total operation costs by model Traditional 2 are all time lower than those by Traditional 1, although the difference is not large, suggesting that cross area distribution has a definite advantage in saving allocation cost, which is of course not surprising from an optimization perspective. Secondly, the two cost curves by Traditional 1 and Traditional 2 behave consistently in their rising and falling trend and arrive at their maximum at the exact same time $t=26$. This is because these two traditional models are based on the same demand forecasting mechanism for the medical resource, and the allocation cost is mainly determined by the allocation volume, that is, the demand. Thirdly, the cost curve generated by our time-space network model is the all time minimum and much lower than that by the two traditional measurements. The allocation cost generated by our model is obvious less than the traditional methods in most of the time. We attribute this significant cost reduction to our proactive forecasting that takes into account the positive impact of

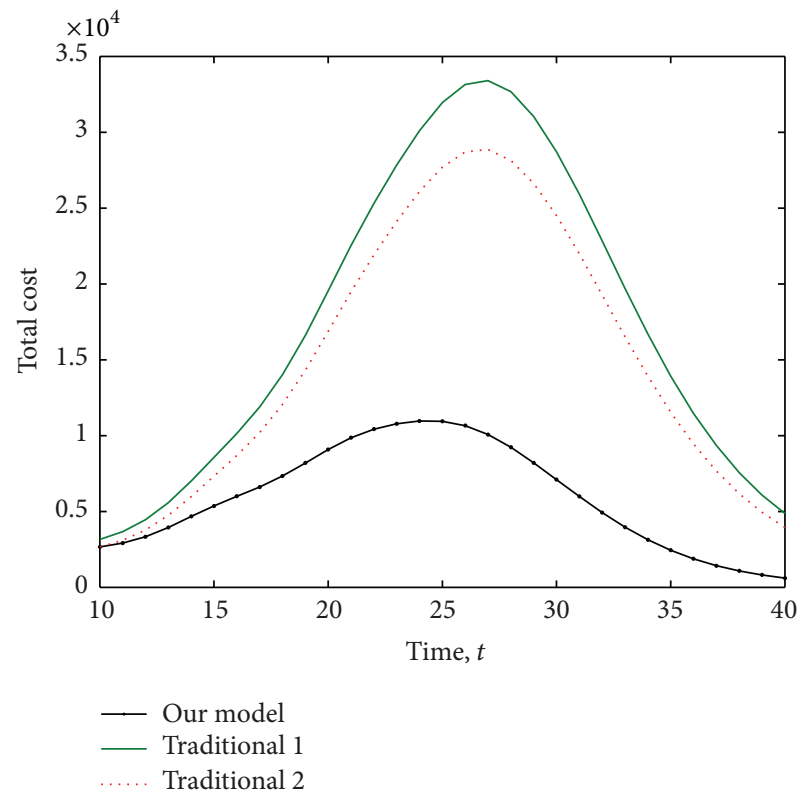

FIgURE 8: Total cost in three different patterns (Unit: \$).

the early allocation of medical resource to the demand in following periods. Finally and most importantly, one can notice that the cost curve by our model reaches its maximum at $t=24.5$, comparing the two traditional measurements at $t=26$. This suggests that by using our proposed model we can get control of the epidemic spread earlier, which stands for an invaluable social merit on top of the economic savings. To conclude our findings in this example, the cross area distribution that features our proposed model and Traditional 2 can reduce the logistic part of the allocation cost. The proactive forecasting model coupled with our time-space optimal allocation programming proposed in this paper can subdue the epidemic diffusion and thus significantly reduce the demand for the medical resource, resulting greater saving in the total operation cost. 


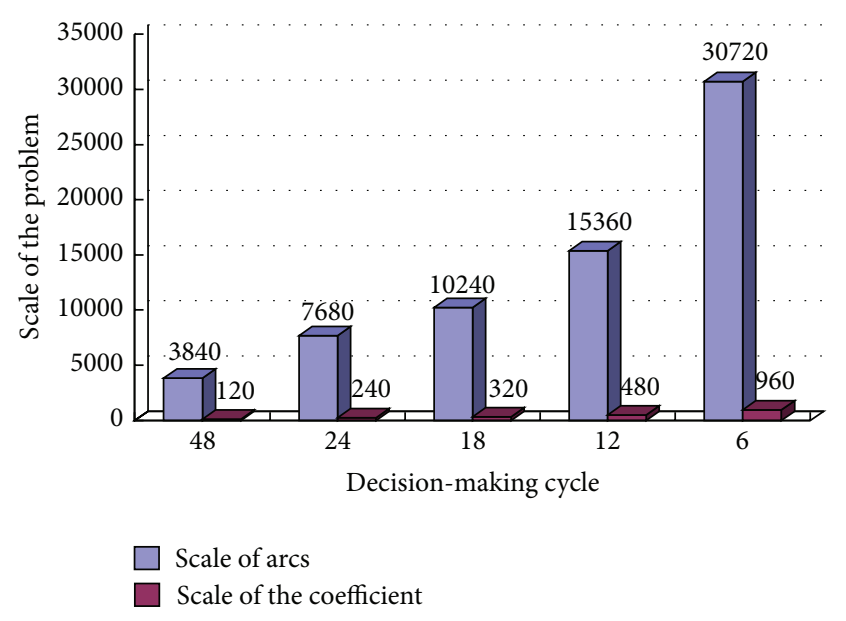

FIGURE 9: Relationship between the scale of problem and decisionmaking cycle.

5.3. Sensitivity Analysis. In this section, a sensitivity analysis of the three key parameters $(\eta, \theta$ and $\Gamma)$ in the timevarying demand forecast model is conducted. According to the definition in Section 3.2, the parameter $\eta$ is closely related to the decision-making cycle. In this paper, the decisionmaking cycle is set to be one day ( 24 hours); so we get a total of $240 \eta$ and 7680 arcs in the experiment. Figure 9 shows the relationship between the scale of the problem and the decision-making cycle (Unit: hour).

In practice, the decision-maker can choose the decisionmaking cycle according to the actual situation. Generally speaking, the shorter the cycle is, the better the forecast accuracy is, but the larger the scale of the problem and its complexity is. On the other hand, if the decision-making cycle is set too short to let the actual distribution operations uncomplete, then the accuracy of the model might be adversely affected. Therefore, the decision-making cycle should be selected appropriately in a practical problem.

As total cost of the proposed medicine logistics network mainly depends on the demand for medical resource, these two variables get a similar variation tendency. Thus, taking EDH1 as an example, we can hold all the parameters fixed, as in the numerical example given in Section 5.1, and let $\theta$ and $\Gamma$ take on five different values, respectively. The demand for medical resource in each decision-making cycle is shown in Figures 10 and 11.

As Figure 10 shows, $\theta$ takes on five values ranging from $60 \%$ to $100 \%$. The larger $\theta$ is, the lower the demand is. Accordingly, the lower the total cost would be. As Figure 11 shows, $\Gamma$ takes on five values ranging from 10 to 20 . The shorter $\Gamma$ is, the lower the demand is, and thus the lower the total cost would be. The above analysis confirms that both of these two key parameters play important roles in medical resource allocation decisions. For a small change of $\theta$ and $\Gamma$, the final allocation decisions and the total operation cost in each cycle can change significantly. Unfortunately, the precise values for these two parameters in an epidemic control are difficult to get. As the accuracy of these two parameters is vital to the success of medicine logistics operation, it calls for more

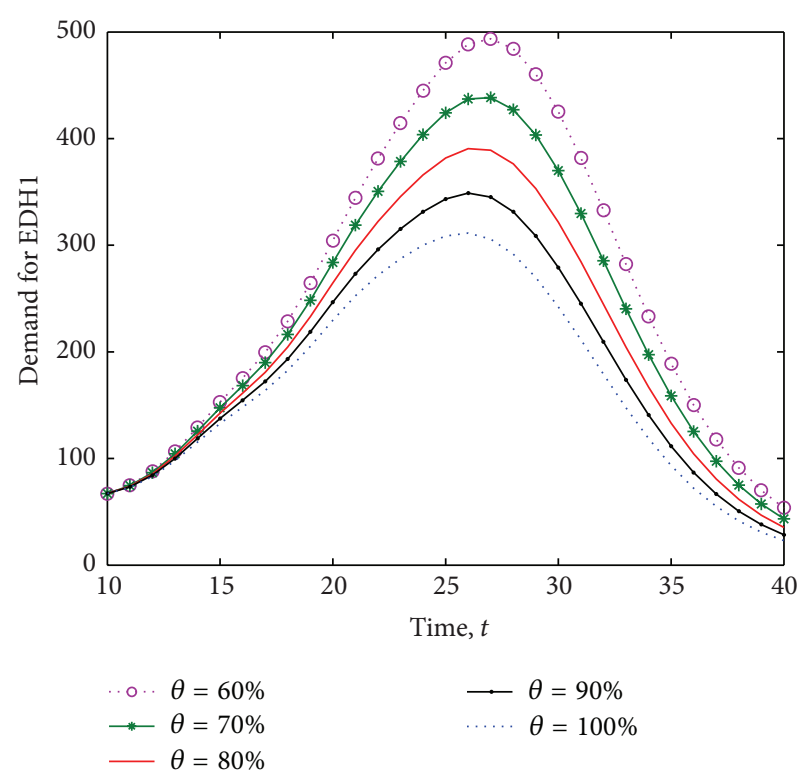

FIGURE 10: Demand in EDH1 with different value of $\theta$.

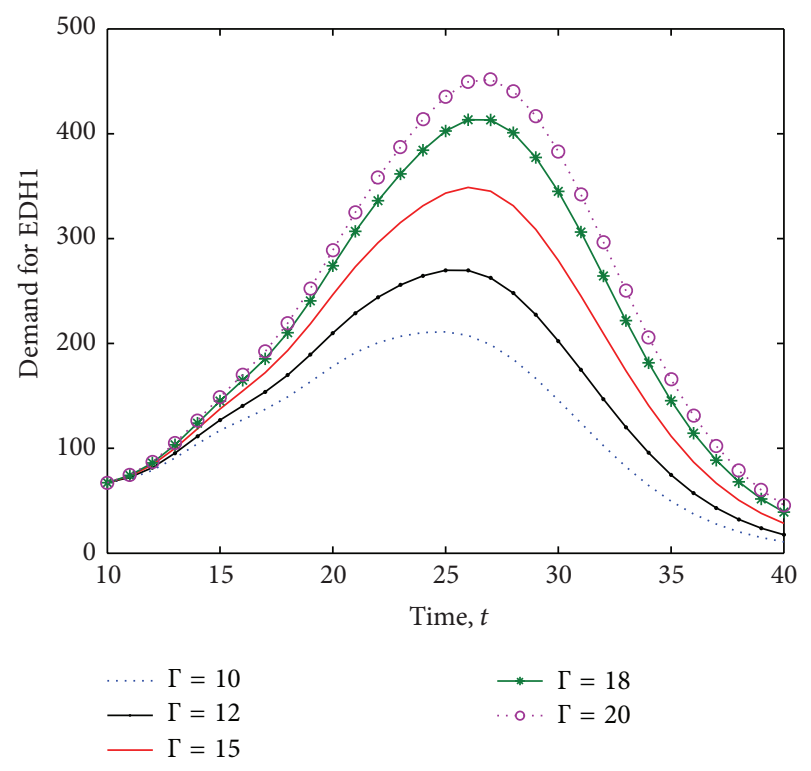

FIGURE 11: Demand in EDH1 with different value of $\Gamma$.

research work to scientifically estimate these two parameters for different epidemics.

\section{Conclusions}

In this paper, we develop a discrete time-space network model to study the medical resource allocation problem in an epidemic outbreak. In each decision-making cycle, the allocation of medical resource across the region from ADCs through DDCs to EDHs is determined by a linear programming model with the dynamic demand that is forecasted by an epidemic diffusion rule. The novelty of our model 
against the existing works in literature is characterized by the following three aspects.

(1) While most research on medical resource allocation studies a static problem which takes no consideration of the time evolution and dynamic nature of the demand, the model proposed in this paper addresses a time-series demand that is forecasted in match of the course of an epidemic diffusion.

(2) The model couples a multistage linear programming for optimal allocation of medical resource with a proactive forecasting mechanism cultivated from the epidemic diffusion dynamics. The two dynamic processes are woven together and interactively proceed to model the epidemic diffusion and the medical resource allocation. The rationale that the medical resource allocated in early periods will take effect in subduing the spread of the epidemic spread and thus impact the demand in later periods has been for the first time incorporated into our model.

(3) The computational results show that the proposed model remarkably outperforms the traditional measurements in both terms of cost reduction and epidemic control. Our model can significantly reduce the total operation cost of the medical resource allocation and may get the epidemic diffusion in control earlier than the traditional measurements.

Furthermore, the medicine logistics operation problem has been decomposed into several mutually correlated subproblems and then been solved systematically in the same decision scheme. Thus, the result will be much more suitable for a real operation. As the limitation of the model, it is developed for the medical resource allocation in a geographic area where an epidemic disease has been spreading and it does not consider possible cross area diffusion between two or more geographic areas. We assume that once an epidemic outbreak exists, the government has effective means to separate the epidemic areas so that cross area spread can be basically prevented. However, this cannot always be guaranteed in reality.

\section{Conflict of Interests}

The authors declare that there is no conflict of interests regarding the publication of this paper.

\section{Acknowledgments}

This work has been partially supported by the National Natural Science Foundation of China (no. 71301076), the MOE Project of Humanities and Social Sciences (no. 11YJCZH109), Natural Science Foundation of Jiangsu Province (BK20130771), and the Research Fund for the Doctoral Program of Higher Education of China (20133219120037). The authors also wish to thank Professor Ding Zhang from State University of New York, Oswego (USA), who read earlier versions of this paper. His helpful suggestions improved the presentation significantly.

\section{References}

[1] J. Hu, A. Z. Zeng, and L. Zhao, "A comparative study of publichealth emergency management," Industrial Management and Data Systems, vol. 109, no. 7, pp. 976-992, 2009.

[2] J. Zhang and Z. Ma, "Global dynamics of an SEIR epidemic model with saturating contact rate," Mathematical Biosciences, vol. 185, no. 1, pp. 15-32, 2003.

[3] B. K. Mishra and D. K. Saini, "SEIRS epidemic model with delay for transmission of malicious objects in computer network," Applied Mathematics and Computation, vol. 188, no. 2, pp. 14761482, 2007.

[4] C. Sun and Y. Hsieh, "Global analysis of an SEIR model with varying population size and vaccination," Applied Mathematical Modelling, vol. 34, no. 10, pp. 2685-2697, 2010.

[5] J. Zhang, J. Li, and Z. Ma, "Global dynamics of an SEIR epidemic model with immigration of different compartments," Acta Mathematica Scientia B: English Edition, vol. 26, no. 3, pp. 551-567, 2006

[6] J. Saramäki and K. Kaski, "Modelling development of epidemics with dynamic small-world networks," Journal of Theoretical Biology, vol. 234, no. 3, pp. 413-421, 2005.

[7] X. Xu, H. O. Peng, X. M. Wang, and Y. H. Wang, "Epidemic spreading with time delay in complex networks," Physica A: Statistical Mechanics and its Applications, vol. 367, pp. 525-530, 2006.

[8] X. P. Han, "Disease spreading with epidemic alert on smallworld networks," Physics Letters A, vol. 365, no. 1-2, pp. 1-5, 2007.

[9] E. Jung, S. Iwami, Y. Takeuchi, and T. Jo, "Optimal control strategy for prevention of avian influenza pandemic," Journal of Theoretical Biology, vol. 260, no. 2, pp. 220-229, 2009.

[10] J. X. Wang, W. S. Xu, R. Q. Zhang, and N. Wu, "Epidemic prevention and infection control of a field dressing station in Wenchuan earthquake areas," International Journal of Infectious Diseases, vol. 13, no. 1, p. 100, 2009.

[11] M. E. Halloran, N. M. Ferguson, S. Eubank et al., "Modeling targeted layered containment of an influenza pandemic in the United States," Proceedings of the National Academy of Sciences of the United States of America, vol. 105, no. 12, pp. 4639-4644, 2008.

[12] K. I. Kim, Z.-G. Lin, and L. Zhang, "Avian-human influenza epidemic model with diffusion," Nonlinear Analysis: Real World Applications, vol. 11, no. 1, pp. 313-322, 2010.

[13] J. Liu and T. Zhang, "Epidemic spreading of an SEIRS model in scale-free networks," Communications in Nonlinear Science and Numerical Simulation, vol. 16, no. 8, pp. 3375-3384, 2011.

[14] M. Samsuzzoha, M. Singh, and D. Lucy, "Numerical study of an influenza epidemic model with diffusion," Applied Mathematics and Computation, vol. 217, no. 7, pp. 3461-3479, 2010.

[15] M. Samsuzzoha, M. Singh, and D. Lucy, "A numerical study on an influenza epidemic model with vaccination and diffusion," Applied Mathematics and Computation, vol. 219, no. 1, pp. 122$141,2012$.

[16] S. Yin, G. Wang, and H. R. Karimi, "Data-driven design of robust fault detection system for wind turbines," Mechatronics, vol. 24, no. 4, pp. 298-306, 2014.

[17] S. Yin, X. Li, H. Gao, and O. Kaynak, "Data-based techniques focused on modern industry: an overview," IEEE Transactions on Industrial Electronics, 2014. 
[18] S. Yin and G. Wang, "Robust PLS approach for KPI related prediction and diagnosis against outliers and missing data," International Journal of Systems Science, vol. 45, no. 7, pp. 1375-1382, 2014.

[19] G. S. Zaric and M. L. Brandeau, "Resource allocation for epidemic control over short time horizons," Mathematical Biosciences, vol. 171, no. 1, pp. 33-58, 2001.

[20] G. S. Zaric and M. L. Brandeau, "Dynamic resource allocation for epidemic control in multiple populations," Journal of Mathematics Applied in Medicine and Biology, vol. 19, no. 4, pp. 235255, 2002.

[21] M. L. Brandeau, G. S. Zaric, and A. Richter, "Resource allocation for control of infectious diseases in multiple independent populations: beyond cost-effectiveness analysis," Journal of Health Economics, vol. 22, no. 4, pp. 575-598, 2003.

[22] G. S. Zaric, D. M. Bravata, J. Cleophas Holty, K. M. McDonald, D. K. Owens, and M. L. Brandeau, "Modeling the logistics of response to anthrax bioterrorism," Medical Decision Making, vol. 28, no. 3, pp. 332-350, 2008.

[23] R. J. Duintjer Tebbens, M. A. Pallansch, J. P. Alexander, and K. M. Thompson, "Optimal vaccine stockpile design for an eradicated disease: application to polio," Vaccine, vol. 28, no. 26, pp. 4312-4327, 2010.

[24] M. Liu and L. Zhao, "Optimization of the emergency materials distribution network with time windows in anti-bioterrorism system," International Journal of Innovative Computing, Information and Control, vol. 5, no. 11A, pp. 3615-3624, 2009.

[25] H. Y. Wang, X. P. Wang, and A. Z. Zeng, "Optimal material distribution decisions based on epidemic diffusion rule and stochastic latent period for emergency rescue," International Journal of Mathematics in Operational Research, vol. 1, no. 1-2, pp. 76-96, 2009.

[26] P. Qiang and A. Nagurney, "A bi-criteria indicator to assess supply chain network performance for critical needs under capacity and demand disruptions," Transportation Research A, vol. 46, no. 5, pp. 801-812, 2012.

[27] N. P. Rachaniotis, T. K. Dasaklis, and C. P. Pappis, "A deterministic resource scheduling model in epidemic control: a case study," European Journal of Operational Research, vol. 216, no. 1, pp. 225-231, 2012.

[28] G. Barbarosolu, L. Özdamar, and A. Çevik, "An interactive approach for hierarchical analysis of helicopter logistics in disaster relief operations," European Journal of Operational Research, vol. 140, no. 1, pp. 118-133, 2002.

[29] S. Y. Yan and Y. L. Shih, "Optimal scheduling of emergency roadway repair and subsequent relief distribution," Computers \& Operations Research, vol. 36, no. 6, pp. 2049-2065, 2009.

[30] M. Liu and L. D. Zhao, "Analysis for epidemic diffusion and emergency demand in an anti-bioterrorism system," International Journal of Mathematical Modelling and Numerical Optimisation, vol. 2, no. 1, pp. 51-68, 2011.

[31] M. Liu, L. Zhao, and H. Sebastian, "Mixed-collaborative distribution mode for emergency resources in an anti-bioterrorism system," International Journal of Mathematics in Operational Research, vol. 3, no. 2, pp. 148-169, 2011.

[32] K. Y. Tham, "An emergency department response to severe acute respiratory syndrome: a prototype response to bioterrorism," Annals of Emergency Medicine, vol. 43, no. 1, pp. 6-14, 2004.

[33] J. B. Sheu, "An emergency logistics distribution approach for quick response to urgent relief demand in disasters," Transportation Research E: Logistics and Transportation Review, vol. 43, no. 6, pp. 687-709, 2007. 


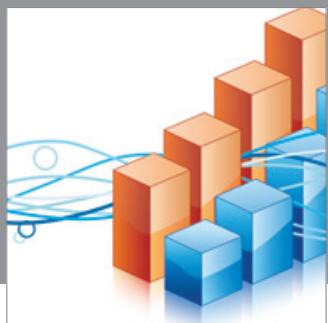

Advances in

Operations Research

mansans

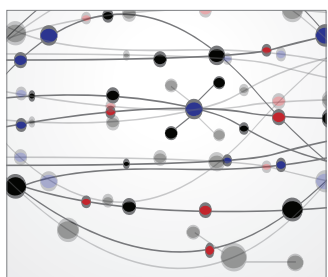

The Scientific World Journal
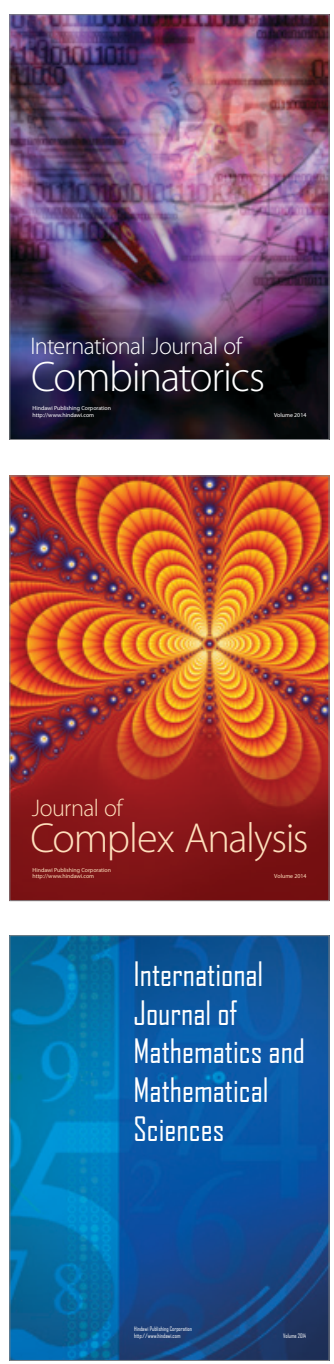
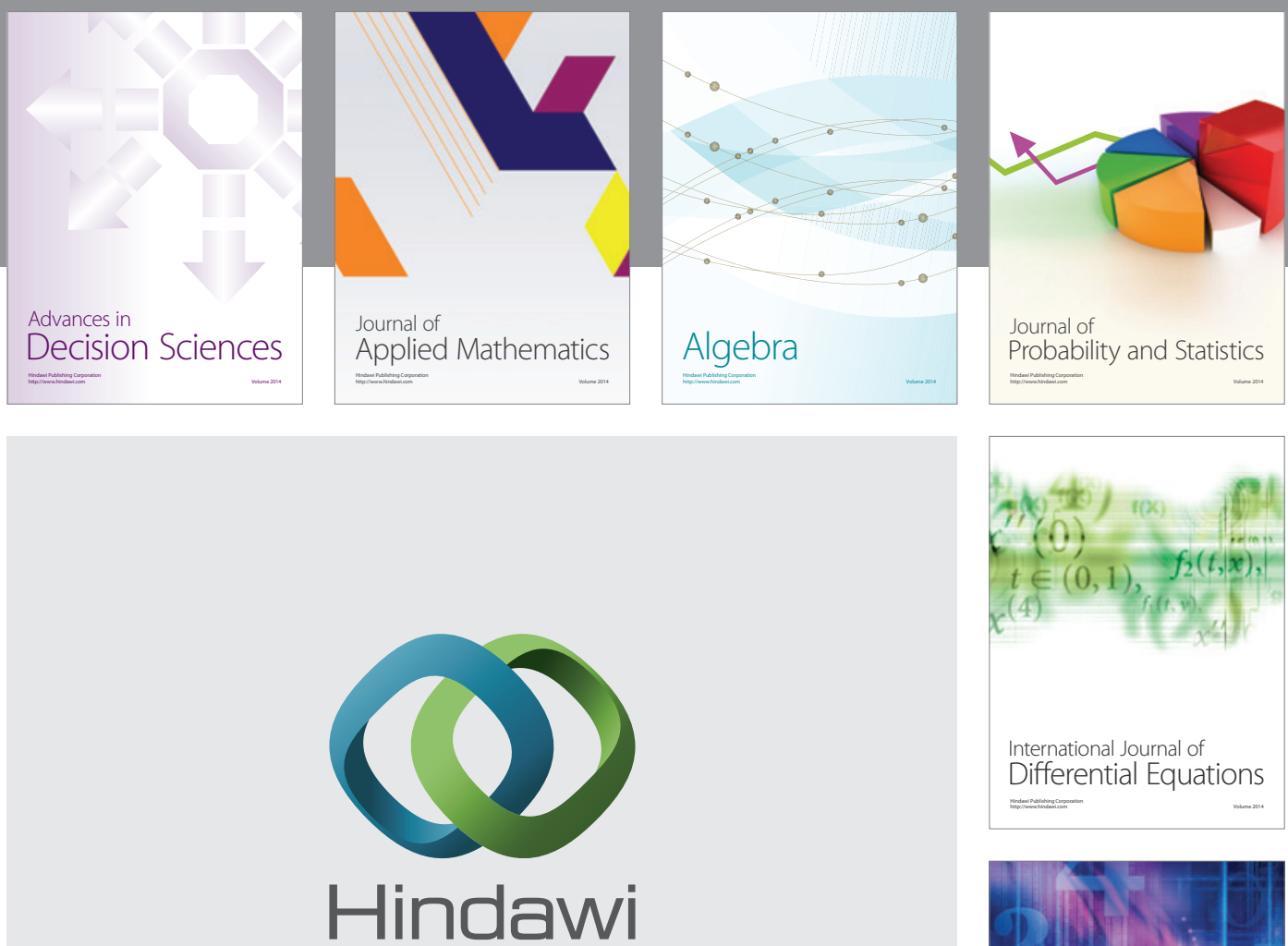

Submit your manuscripts at http://www.hindawi.com
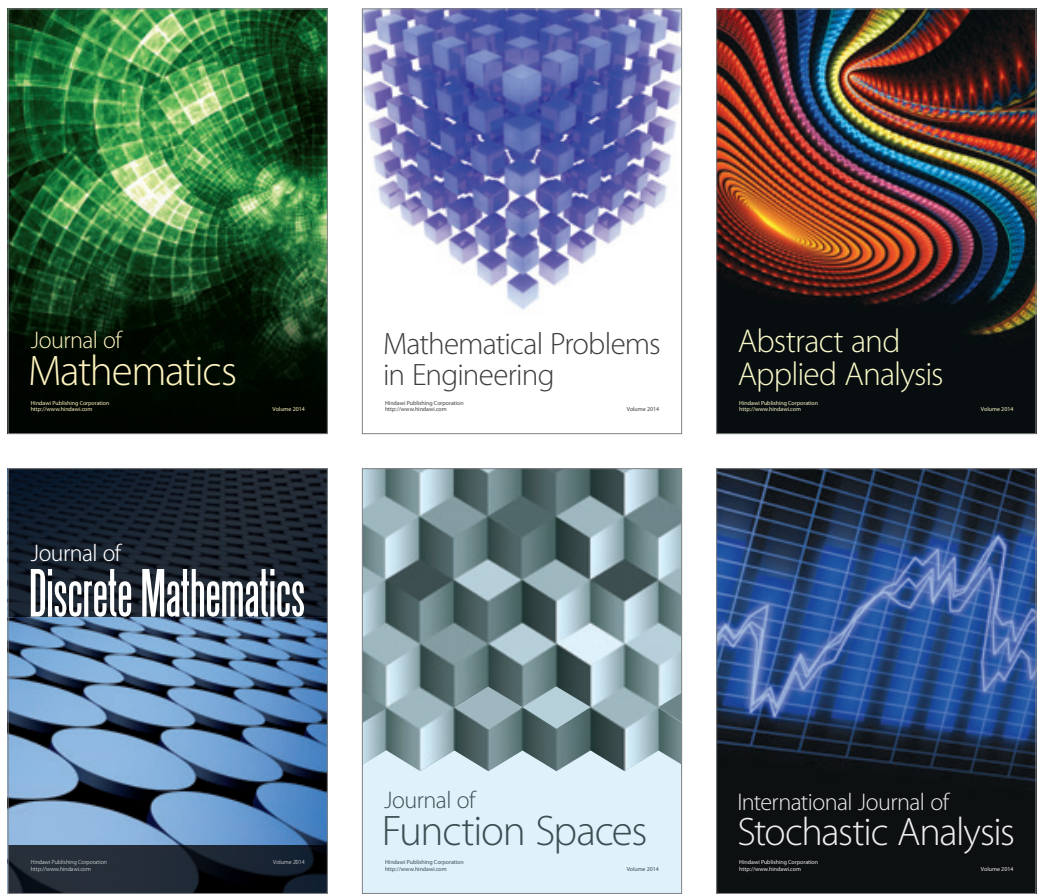

Journal of

Function Spaces

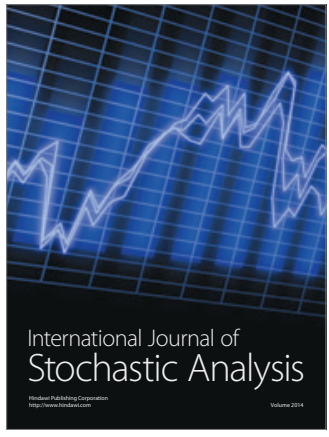

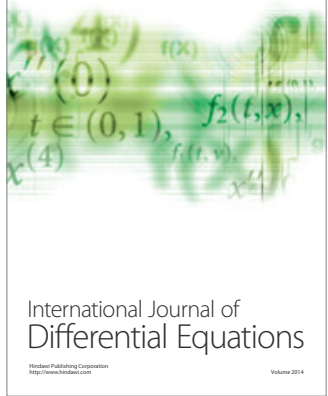
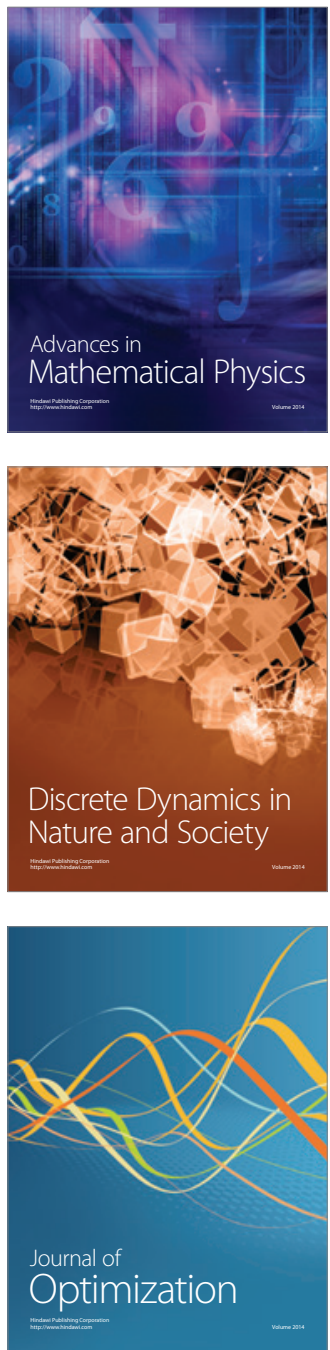